\title{
Taquiarritmias Supraventriculares no Feto. Experiência de uma Unidade de Referência em Cardiologia Fetal
}

\author{
Paulo Zielinsky, Rejane F. Dillenburg, G ustavo G. de Lima, Lúcia P. Zimmer \\ PortoAlegre, RS
}

Objetivo - Avaliar a forma de apresentação, diagnóstico e tratamento das taquiarritmias supraventriculares fetais, através do relato de uma série de casos acompanhados em um centro terciário de cardiologia fetal.

Métodos - São descritos 25 casos de taquiarritmia supraventricular diagnosticados intra-útero, no período de janeiro/89 a outubro/97, em uma população compreendendo 3117 gestantes.

Resultados - Foram diagnosticados 17 casos de taquiarritmia supraventricular e 8 casos de flutter atrial fetal. As idades gestacionais variaram de 26 a 40 semanas. Doze fetos apresentavam hidropisia no momento do diagnóstico (6 com taquicardia supraventricular (TSV) $e$ 6 com flutter atrial). Quatro fetos com TSV apresentavam cardiopatias estruturais (dois casos de anomalia de Ebstein e dois com comunicação interventricular). Todos os fetos foram internados na Unidade de Cardiologia Fetal para monitorização e tratamento. Entre os 17 fetos com TSV, 12 apresentaram reversão da arritmia após administração de digoxina, mas esta medida não foi eficaz em nenhum paciente com flutter. Dois pacientes com TSV e seis com flutter necessitaram interrupção da gestação para cardioversão elétrica pós-natal. A mortalidade foi de 3/17 no grupo da TSV (incluindo dois pacientes com anomalia de Ebstein) e de 0/8 no grupo com flutter.

Conclusão - As taquiarritmias supraventriculares fetais são eventos raros na população geral. Entretanto, podem provocar insuficiência cardíaca e óbito intrauterino. Como a resposta ao tratamento é satisfatória, tornam-se de extrema importância o diagnóstico precoce e o tratamento adequado.

Palavras-chave: arritmias, coração fetal, ecocardiografia

\section{Fetal Supraventricular Tachyarrhythmias. Experience of a Fetal Cardiology Reference Center}

\begin{abstract}
Purpose - To describe the presentation, diagnosis and treatment of fetal supraventricular tachyarrhythmias in a series offetuses followed in a tertiary Fetal Cardiology Center.

Methods - Twenty-five fetuses with diagnosis of supraventricular tachyarrhytmia were reported from January 1989 to October 1997, among 3117 pregnant women referred for fetal cardiac evaluation.

Results - There were 17 fetuses with the diagnosis of supraventricular tachycardia (SVT) and 8 patients with atrial flutter $(A F)$. Gestational age ranged from 26 to 40 weeks. Twelve patients were hydropic at presentation (6 with SVT and $6 A F$ ). Four fetuses with SVT showed structural abnormalities (two with Ebsten's anomaly and two with VSD). All patients were admitted to the Fetal Cardiology Unit for monitoring and treatment. Among 17 fetuses with SVT, twelve showed good response to digoxin administration, but this drug was not useful in any of the patients with flutter. In two patients with SVT and in six with AF, the pregnancy was interrupted to perform post-natal cardioversion. The mortality rate was $3 / 17$ in the SVT group (including 2 patients with ebstein's anomaly and $0 /$ 8 in the flutter group)

Conclusion - Fetal supraventricular tachyarrithmias are rare in the general population. Nevertheless, the fetus may present with severe heart failure and death. Considering the satisfactory therapeutic response, accurate diagnosis and early treatment of these conditions are extremely important.
\end{abstract}

Key-words: fetal heart, arrhythmias, echocardiography

Arq Bras Cardiol, volume 70 (n 5), 337-340, 1998

A detecção de alterações do ritmo cardíaco fetal pode ocorrer em fase relativamente precoce da gestação ${ }^{1}$. Apesar da baixa prevalência das taquiarritmias fetais $(0,4-0,6 \%)^{2}$,é

Unidade de Cardiologia Fetal do Instituto de Cardiologia do Rio Grande do Sul Correspondência: Paulo Zielinsky - Av Princesa Isabel, 395 - 90620-001 - Porto Alegre, RS

Recebido para publicação em 21/1/98

Aceito em 11/3/98 importante diferenciar as taquiarritmias benignas (ritmo sinusal, de início gradual, intermitente e associada à movimentação fetal) daquelas que necessitam tratamento específico e acompanhamento cuidadoso da sua evolução, para detecção precoce de sinais de insuficiência $\operatorname{cardíaca}^{3}$ (IC). Os sinais de IC podem se desenvolver rapidamente no feto, evoluindo a partir de efusões pericárdicas, pleurais, edema subcutâneo e atingindo o grau máximo na hidropisia fetal. 
Portanto, as taquiarritmias que apresentam início súbito, freqüência cardíaca (FC) acima de 200bpm, mesmo que não sustentadas, merecem investigação ${ }^{3,4}$. O diagnóstico é feito através do ecocardiograma fetal, com a identificação da seqüência temporal entre a sístole atrial e ventricular, sendo seu registro realizado no modo $\mathrm{M}$ e mensurado em milissegundos ${ }^{3-7}$. As opções de tratamento incluem o uso de drogas por via materna (transplacentária) ou fetal direta (cordocentese) ${ }^{8}$. Nos casos refratários ao tratamento medicamentoso, indica-se o parto para manejo da arritmia no período neonatal, quando a maturidade pulmonar estiver presente $^{9,10}$.

\section{Métodos}

Foram revisados os registros médicos de 3117 gestantes encaminhadas para um centro terciário de cardiologia fetal, de janeiro/89 a outubro/97, onde foram diagnostica$\operatorname{dos} 25(0,83 \%)$ casos de taquiarritmias fetais. Todas as gestantes foram internadas para diagnóstico e tratamento do feto. O diagnóstico baseou-se na análise da seqüência de contração atrial e ventricular ao ecocardiograma unibidimensional. O ritmo foi considerado sinusal quando a uma contração atrial seguia-se imediatamente uma contração ventricular, caracterizando condução atrioventricular (AV) normal. A taquicardia supraventricular (TSV) foi definida como um ritmo de freqüência $>200 \mathrm{bpm}$, com pequena variabilidade batimento a batimento, de início e término súbitos, sustentado ou não, com condução AV 1:1. O flutter foi definido como um ritmo com freqüiência atrial de 300-460, regular, com ou sem dissociação $\mathrm{AV}^{4,7}$. Todos os pacientes foram avaliados através de ecocardiogramas seriados. Definido o mecanismo da taquiarritmia, a instituição do tratamento dependem basicamente das condições hemodinâmicas do feto e do grau de maturidade pulmonar fetal, avaliado pela análise da relação L:E (lecitina: esfingo- mielina) do líquido amniótico. As drogas utilizadas foram administradas inicialmente por via oral materna, ou por cordocentese, nos casos refratários ao tratamento transplacentário. As medicações empregadas foram digoxina, amiodarona, adenosina e sotalol. O objetivo inicial do tratamento era reversão ao ritmo sinusal intra-útero ou, nos casos de evolução menos favorável, o controle da freqüência ventricular com redução dos sinais de IC fetal. A indicação de parto para tratamento pós-natal dependeu da resposta fetal às tentativas de cardioversão química.

\section{Resultados}

Foram diagnosticados 17 casos de TSV e oito casos de flutter atrial (tab. I). A principal indicação para a realização da ecocardiografia fetal foi a presença de arritmias ao exame obstétrico em oito e quatro casos, respectivamente. Nos casos de TSV (tab. II), as idades gestacionais variaram de 21 a 38 semanas, no momento do diagnóstico. A FC variou entre 200 e $280 \mathrm{bpm}$. Este grupo incluiu uma gestação gemelar em que apenas um dos fetos apresentava arritmia. Entre os fetos com TSV, foram observados dois casos de anomalia de Ebstein da valva tricúspide, um deles acompa-

\begin{tabular}{|lcc|}
\hline \multicolumn{3}{|c|}{ Tabela I - Casos de taquiarritmia supraventricular } \\
& TSV & Flutter atrial \\
\hline $\mathrm{n}$ & 17 & 8 \\
Idade gestacional & $21-38$ semanas & $29-40$ semanas \\
& média 27,8 & média 34,2 \\
Freqüência cardíaca(bpm) & $200-280$ & $160-350$ \\
Condução AV & $1: 1$ & $7(2: 1) / 1(1: 1)$ \\
Hidropisia & $6 / 17$ & $6 / 8$ \\
Ritmo sinusal intra-útero & $13 / 17$ & $2 / 8$ \\
Parto para cardioversão & $2 / 17$ & $6 / 8$ \\
Mortalidade fetal & $2 / 17$ (anomalia de Ebstein) & $0 / 8$ \\
Mortalidade neonatal & $1 / 17$ (prematuridade) & $0 / 8$ \\
\hline TSV- taquicardia supraventricular; AV- atrioventricular. & \\
\hline
\end{tabular}

\begin{tabular}{|c|c|c|c|c|c|c|}
\hline & $\begin{array}{c}\text { Idade } \\
\text { gestacional }\end{array}$ & $\begin{array}{l}\text { Frequiência } \\
\text { cardíaca }\end{array}$ & Hidropisia & $\begin{array}{c}\text { Cardiopatia } \\
\text { estrutural }\end{array}$ & $\begin{array}{c}\text { Medidas } \\
\text { terapêuticas }\end{array}$ & Evolução \\
\hline 1 & 26 & 280 & $\operatorname{sim}$ & CIV & digoxina VM & RS IU \\
\hline 2 & 28 & 230 & não & não & digoxina VM & RS IU \\
\hline 3 & 32 & 210 & não & não & digoxina VM & RS IU \\
\hline 4 & 30 & 240 & $\operatorname{sim}$ & não & digoxina adenosina amiodarona $\mathrm{VM}+\mathrm{C}$ & CVE pós-parto RS \\
\hline 5 & 31 & 210 & não & não & digoxina VM & RS IU \\
\hline 6 & 32 & 200 & $\operatorname{sim}$ & não & digoxina VM & RS IU \\
\hline 7 & 30 & 210 & não & não & digoxina VM & RS IU \\
\hline 8 & 30 & 260 & $\operatorname{sim}$ & não & digoxina adenosina amiodarona $\mathrm{VM}+\mathrm{C}$ & CVE pós-parto RS óbito por PMT \\
\hline 9 & 37 & 260 & não & não & digoxina VM & RS IU \\
\hline 10 & 30 & 220 & não & não & digoxina VM & RS IU \\
\hline 11 & 35 & 250 & não & não & digoxina VM & RS IU \\
\hline 12 & 38 & 240 & não & não & digoxina VM & RS IU \\
\hline 13 & 29 & 220 & não & não & digoxina VM & RS IU \\
\hline 14 & 29 & 200 & $\operatorname{sim}$ & Ebstein com IT severa + EP & digoxina VM diuréticos $\mathrm{VM}+\mathrm{C}$ & RS IU + ESSV, óbito fetal \\
\hline 15 & 21 & 210 & não & DVSVD & digoxina VM & RS IU \\
\hline 16 & 30 & 210 & $\operatorname{sim}$ & Ebstein & digoxina diuréticos $\mathrm{VM}+\mathrm{C}$ & RS IU óbito fetal \\
\hline 17 & 34 & 200 & não & não & digoxina sotalol & $\mathrm{RS} / \mathrm{U}$ \\
\hline
\end{tabular}




\begin{tabular}{|c|c|c|c|c|c|c|c|c|}
\hline \multicolumn{9}{|c|}{ Tabela III - Casos de flutter atrial } \\
\hline $\mathrm{N}^{\circ}$ & IG & FA & $\mathrm{FV}$ & BAV & Hidropisia & Tratamento & Resultados & MC \\
\hline 1 & 36 & 440 & 220 & $2: 1$ & não & digoxina parto CVE & $\mathrm{RS} / \mathrm{PN}$ & não \\
\hline 2 & 36 & 400 & 200 & $2: 1$ & $\operatorname{sim}$ & procainamida amiodarona parto CVE & $\mathrm{RS} /$ flutter/RS/PN & não \\
\hline 3 & 33 & 450 & 250 & $2: 1$ & $\operatorname{sim}$ & parto CVE & $\mathrm{RS} / \mathrm{PN}$ & não \\
\hline 4 & 40 & 450 & 230 & $2: 1$ & sim & digoxina parto CVE & $\mathrm{RS} / \mathrm{PN}$ & não \\
\hline 5 & 35 & $380-440$ & $190-220$ & $2: 1$ & $\operatorname{sim}$ & parto CVE & $\mathrm{RS} / \mathrm{PN}$ & não \\
\hline 6 & 31 & 450 & 240 & $2: 1$ & sim & digoxina amiodarona & RS & não \\
\hline 7 & 29 & 330 & 330 & $1: 1$ & $\operatorname{sim}$ & digoxina amiodarona & RS & não \\
\hline 8 & 35 & 440 & 220 & $2: 1$ & não & digoxina sotalol parto CVE & $\mathrm{RS} / \mathrm{PN}$ & não \\
\hline
\end{tabular}

nhado de estenose pulmonar, e duas comunicações interventriculares (CIV).

Nos 17 fetos com TSV, a hidropisia fetal estava presente em seis. Destes, três apresentavam cardiopatias congênitas (dois casos de anomalia de Ebstein e um CIV). Todos os pacientes receberam inicialmente digoxina por via materna. Todos os fetos não-hidrópicos tiveram resposta adequada a esta medida terapêutica, com reversão para ritmo sinusal e progressão da gestação sem intercorrências. Entre os seis fetos hidrópicos, por outro lado, em apenas dois houve sucesso terapêutico após administração de digoxina por via transplacentária, com reversão a ritmo sinusal intra-útero. Um deles era portador de uma CIV pequena. Ambos mantiveram-se em ritmo sinusal após o nascimento.

Outros dois pacientes necessitaram de administração direta de drogas (incluindo digoxina, adenosina e amiodarona), através de cordocentese e interrupção da gestação para cardioversão elétrica. Estes dois fetos tinham 30 semanas de idade gestacional na apresentação, com FC variando entre 240 e $260 \mathrm{bpm}$, sem malformações cardíacas associadas. $\mathrm{O} 1^{\circ}$ nasceu com 33 semanas, tendo boa resposta à cardioversão elétrica pós-natal e $\mathrm{o} 2^{\circ}$ recebeu amiodarona e adenosina por cordocentese, sem resposta. Nasceu com 30 semanas de idade gestacional, sendo revertido a ritmo sinusal após o parto. Este bebê evoluiu para óbito após alguns dias, por complicações da prematuridade.

Os dois fetos hidrópicos com TSV e anomalia de Ebstein evoluíram para óbito ainda no período pré-natal.

Em apenas um caso foi observada presença de pré-excitação ventricular no eletrocardiograma pós-natal.

Dentre os oito casos de flutter (tab. III), com idades gestacionais entre 31 e 40 semanas na apresentação, seis estavam hidrópicos no momento do diagnóstico. As freqüências atriais variaram de 340 a 440, sendo que sete apresentavam bloqueio atrioventricular (BAV) 2:1, e um caso apresentava condução AV 1:1. Nenhum feto deste grupo tinha lesão cardíaca estrutural. Em cinco casos foi necessária a interrupção da gestação para cardioversão elétrica pósnatal com reversão a ritmo sinusal. Em dois casos, foi indicado tratamento com amiodarona por cordocentese devido à refratariedade ao tratamento transplacentário. Um destes pacientes, com 31 semanas de idade gestacional, recebeu inicialmente digoxina, sem resposta. Foi administrada amiodarona por cordocentese na dose de $15 \mathrm{mg} / \mathrm{kg}$ de peso estimado. Houve resposta adequada e reversão a ritmo sinusal. Entretanto, houve recidiva da taquiarritmia, que foi adequadamente revertida após nova infusão intra-umbilical de amiodarona, associada à administração materna da droga. Após alguns dias de tratamento, observou-se melhora dos sinais de hidropisia. $\mathrm{Na}$ alta hospitalar, o ritmo era sinusal, mantendo-se assim desde as 35 semanas de idade gestacional até o termo da gestação. O outro feto estava com 25 semanas de idade gestacional, na apresentação. $\mathrm{O}$ ecocardiograma uni-bidimensional demonstrou a presença de taquiarritmia supraventricular com condução AV 1:1. O feto não estava hidrópico, no momento do diagnóstico, e o coração era estruturalmente normal. Foi administrada digoxina por via oral materna. No $3^{\circ}$ dia de tratamento houve piora da IC fetal, com derrame pericárdico, pleural e ascite. A administração de adenosina por cordocentese determinou uma queda da FC de 340 para $170 \mathrm{bpm}$, secundária ao desenvolvimento de BAV 2:1, estabelecendo, assim, o diagnóstico de flutter atrial. A seguir foi iniciado o tratamento com amiodarona $(15 \mathrm{mg} / \mathrm{kg}$ ) via cordocentese, com interrupção da arritmia. Houve recorrência do flutter, que respondeu a associação de digoxina e amiodarona por cordocentese e via materna. No $15^{\circ}$ dia de tratamento, foi suspensa a digoxina, e mantida a amiodarona na dose materna de 200mg/dia. Na alta hospitalar, com 29 semanas de gestação, o ritmo era sinusal. Em ambos os casos, foram realizadas provas laboratoriais de função tireoidiana, que foram normais.

\section{Discussão}

A prevalência de taquiarritmias fetais em populações não selecionadas é de $0,4-0,6 \%{ }^{2}$. Nesta série, a prevalência foi de $0,83 \%$, provavelmente porque as gestantes eram encaminhadas por suspeita de anormalidade no ritmo cardíaco, a partir do exame obstétrico, constituindo uma população submetida a uma triagem prévia.

Geralmente é descrita uma baixa prevalência de malformações cardíacas associadas nos fetos com taquiarritmias ${ }^{11}$. Nesta série, no entanto, foram observados quatro casos de cardiopatia estrutural, todos no grupo da TSV.

As taquiarritmias fetais podem ser classificadas em 
dois grupos, dependendo da condução AV: taquicardias com condução AV 1:1 e taquicardias com dissociação AV. As taquicardias com condução 1:1 incluem as taquicardias por reentrada $\mathrm{AV}$, taquicardia por reentrada nodal $\mathrm{AV}$ ou, menos comumente, taquicardia por reentrada intra-atrial. Quando há dissociação AV, os mecanismos mais prováveis são a reentrada intra-atrial e a taquicardia atrial automática ${ }^{5}$.

Nos casos de TSV, as idades gestacionais dos fetos foram menores do que nos casos de flutter. Observou-se maior proporção de hidropisia nos pacientes com flutter, apesar de freqüências ventriculares serem semelhantes nos dois grupos. Uma possível explicação é que a contração atrial efetiva seja muito importante para a manutenção do débito cardíaco no feto, já que a complacência ventricularé menor, sendo o volume de ejeção dependente da fase de enchimento tardio, que ocorre com a sístole atrial. Nos casos de flutter, a freqüência atrial é muito alta, impedindo a sístole atrial efetiva e prejudicando o enchimento ventricular adequado, ocasionando IC.

O tratamento transplacentário das taquiarritmias fetais é iniciado com digoxina, droga usualmente eficaz nos fetos não-hidrópicos ${ }^{11-14}$. A digoxina isolada foi eficaz em 12 dos 17 casos de TSV, mas não teve resultado em nenhum caso de flutter. Em um caso de TSV após o tratamento com digoxina houve reversão para ritmo sinusal, mas houve recorrência da arritmia e, no $2^{\circ}$ episódio, foi necessária a utilização de sotalol para reversão e manutenção de ritmo sinusal. Nesta série, 6/17 fetos com TSV e 6/8 fetos com flutter atrial apresentaram-se hidrópicos. A resposta ao uso de digoxina transplacentária, com reversão a ritmo sinusal intra-útero, nos casos de TSV, ocorreu na proporção de dois em seis fetos hidrópicos. Dois pacientes necessitaram cardioversão elétrica pós-natal; os outros dois pacientes com hidropisia eram portadores de anomalia de Ebstein e, apesar da reversão para ritmo sinusal, evoluíram para óbito intra-útero. Nos casos de flutter, dos seis pacientes hidrópicos, dois necessitaram, além da digoxina, da administração direta de amiodarona para reversão a ritmo sinusal intra-útero, enquanto quatro necessitaram indução do parto para cardioversão pós-natal.
O tratamento fetal com amiodarona tem sido bem tolerado pela mãe, provavelmente em função da curta duração de uso da droga. Dentre os efeitos adversos possíveis com tratamentos de curta duração figura o hipotireoidismo, descrito após duas semanas de uso ${ }^{13}$. O feto e o recém-nascido são mais sensíveis a sobrecargas de iodo. Nesta série, foi realizada a monitorização da função tireoidiana durante o uso da amiodarona, não tendo sido detectado nenhum caso de hipotireoidismo fetal.

Geralmente o tratamento é mantido até o parto. Existem riscos potenciais com o uso de amiodarona durante anestesia regional utilizada para a cesariana ${ }^{15}$ : hipotensão arterial severa devido aos efeitos combinados do bloqueio simpático e ausência de resposta vasoconstritora secundária ao uso de amiodarona; e além disso, a amiodarona potencializa a diminuição da condução intracardíaca e contratilidade determinada pelos anestésicos locais. Este último efeito não foi descrito com anestesia epidural.

A adenosina é um nucleosídeo do grupo das purinas que age através de um receptor específico causando diminuição da freqüência sinusal e bloqueio da condução do nó AV. Tem sido utilizada como agente diagnóstico e terapêutico. No caso de flutter, com condução AV 1:1, o diagnóstico diferencial incluiu TSV com freqüência atrial de $330 \mathrm{bpm}$. Neste caso, o uso de adenosina provocou dissociação AV, confirmando a hipótese de flutter atrial.

Outros agentes citados incluem a procainamida, a quinidina, o propanolol, e a flecainida ${ }^{11}$.

Na literatura, a incidência de complicações decorrentes da cordocentese é de 0,5-1\%. Nesta série, não ocorreu nenhuma complicação deste procedimento. Considerando o baixo risco da cordocentese, e o alto risco de morbidade e mortalidade nos casos de fetos hidrópicos com imaturidade pulmonar, o tratamento agressivo da TSV com amiodarona via cordocentese surge como uma opção terapêutica útil.

Esta série de casos descreve a evolução intra-útero e pós-natal imediata das taquiarritmias fetais. Um dos objetivos a médio prazo é acompanhar essas crianças durante os primeiros anos de vida e avaliar a evolução das anormalidades do ritmo cardíaco presentes na vida fetal.

\section{Referências}

1. Meijboom EJ, van Engelen Ad, van de BeekEW, Weijteins O, Lautenschutz JM, Benatar AA - Fetal arrhythmias. Current Opinion in Cardiology 1994, 9: 97-102.

2. Allan L - Fetal Arrhythymias. In: Long WA. Fetal and Neonatal Cardiology Londres: WB Saunders, 1989; 17: 180-4.

3. Zielinsky P - Arritmias cardíacas fetais: papel da ecocardiografia pré-natal no diagnóstico e na terapêutica intra-uterina. In: Cruz FES, Maia IG. Eletrofisiologia Clínica e Intervencionista das Arritmias Cardíacas. Rio de Janeiro: Ed.Revinter 1997: 317-23.

4. Friedman AH, Copel JA, Kleinman CS - Fetal echocardiography and fetal cardiology: indications, diagnosis and management. Semin Perinatol 1993; 17: 76-88.

5. Naheed ZJ, Strasburger JF, Deal BJ, Benson DW, Gidding SS - Fetal tachycardia: mechanisms and predictors of hydrops fetalis. J Am Coll Cardiol 1996; 27 : 1736-40.

6. van Engelen AD, Weijtens O, Brenner JI, Kleinman CS, Copel JA, Stoutenbeek P - MeijboomE. Management outcome and follow up of fetal tachycardia. J Am Coll Cardiol 1994; 24: 1371-5.

7. Bergmans MGM, Jonker GJ, Kock HCLV - Fetal supraventricular tachycardia Review of the literature. Obstet Gynecol Surv 1985; 40: 61-8.
8. Ito S, Magee L, Smallhorn J - Drug therapy for fetal arrhythmias. Clinics in Perinatology 1994; 21: 543-72.

9. Kleinman CS, Copel JA - Electrophysiological principles and fetal antiarrhythmic therapy. Ultrasound Obstet Gynecol 1991: 286-97.

10. Benisty AA, Algrain J, Guirgis NM, Decrepy A, Cury JF, Blot P - Clinical and pharmacologic study of fetal supraventricular tachyarrhythmias. J Pediatr 1992; 121: 608-13.

11. Simpson LL, Marx GR - Diagnosis and treatment of structural fetal cardiac abnormality and dysrhythmia. Semin Perinatol 1994; 18: 215-27.

12. Soyeur DJ - Atrial flutter in the human fetus: diagnosis, hemodynamic consequences and therapy. J Cardiovasc Electrophysiol 1996; 7: 989-98.

13. Ito $S$, Magee L, Smalhorn J - Drug Therapy for fetal arrhythmias. Clinics Perinatol 1988; 21: 543-72.

14. Weiner CP, Thompson MB - Direct treatment of fetal supraventricular tachycardia after failed transplacental therapy. Am J Obstet Gynecol 1988; 158: 570-3.

15. Fulgencio JP, Hamza J - Anaesthesia for Caesarean section in a patient receiving high dose amiodarone for fetal supraventricular tachycardia. Anaesthesi* 1994; 49: 406-8. 